\title{
Motivaciones y hábitos de actividad física en alumnos universitarios Motivations and physical activity habits in university students
}

\author{
Javier Rico-Díaz, Constantino Arce-Fernández, Alexis Padrón-Cabo, Lucía Peixoto-Pino, Cristian Abelairas-Gómez
}

Universidad de Santiago de Compostela (España)

\begin{abstract}
Resumen. El objetivo de este estudio ha sido analizar los hábitos y motivaciones de práctica de actividad física-deportiva del alumnado universitario. En el estudio participó una muestra de 608 universitarios del campus de Santiago de Compostela, seleccionados por muestreo aleatorio estratificado. Para el análisis de datos se establecieron dos grupos (hombres vs. mujeres) y se realizaron comparaciones por medio de los test chi-cuadrado y t de Student, situando el nivel de significación en $p<.05$. El 74.35\% de los universitarios practicaban algún tipo de actividad físico-deportiva, y el 34.38\% de los encuestados afirmó practicar con una frecuencia de tres o más veces por semana. La edad media de abandono de la práctica físico-deportiva se situó en los 18.42 años. Hacer ejercicio físico fue la principal motivación entre los estudiantes que practicaban alguna actividad física o deportiva. El motivo más señalado para el abandono de la actividad física fue la falta de tiempo para estudiar.
\end{abstract}

Palabras clave. Deporte, ejercicio físico, estudiantes universitarios, abandono, estilo de vida, salud.

\begin{abstract}
The aim of this study was to analyze habits and motivation towards practicing physical-sports activities among university students. A sample of 608 university students from the Santiago de Compostela campus, selected by stratified random sampling, participated in the study. For data analysis, two groups (men vs. women) were established and comparisons were made by means of the chi-square and t-tests of Student, placing the level of significance at $p<.05$. Results display that $74.35 \%$ of college students practiced some type of physical-sports activities, and $34.38 \%$ of respondents claimed that they practiced three or more times per week. The average age of abandonment of physical-sports practice was 18.42 years old. Physical exercise was the main motivation among students who practiced some physical or sports activities. The most important reason for abandoning physical activity was the lack of time for studying.
\end{abstract}

Keywords. Sports, physical exercise, university students, abandonment; lifestyle; health.

\section{Introducción}

Según la Organización Mundial de la Salud (2010) la pérdida de un estilo de vida activo constituye el cuarto factor de riesgo de mortalidad en todo el mundo, provocando aproximadamente un $6 \%$ de las defunciones a nivel global, sin tener en cuenta las enfermedades transmisibles. La literatura científica ha denominado a este fenómeno como «Síndrome de Muerte por Sedentarismo» (Lees \& Booth, 2004). Los efectos del sedentarismo poseen una mayor magnitud en países con un elevado grado de desarrollo (Powell \& Blair, 1994). La inactividad física incrementa las posibilidades de contraer enfermedades cardiovasculares, accidentes cerebrovasculares, hipertensión, diabetes tipo II, cáncer de colon, cáncer de mama y osteoporosis (Garrett, Brasure, Schmitz, Shultz \& Huber, 2004). Además, también ha sido descrito que la práctica de actividad física está estrechamente relacionada no solo con la salud física, sino también con una mejor salud psicológica y calidad de vida (HerreraGutiérrez, Olmos-Soria \& Brocal-Pérez, 2015). Por ello, la implementación de políticas relacionadas con un incremento de los niveles de actividad física en la población podría suponer un factor estratégico para la mejora de la salud y del bienestar físico y mental (Bull et al., 2010).

En el ámbito universitario, la practica físico-deportiva parece tener efectos beneficiosos sobre la mejora de estrategias de enfrentamiento de los estudiantes, de la autoestima, del patrón cognitivo de autopercepción (Joseph, Royse, Benitez \& Pekmezi, 2014), y de las capacidades para el establecimiento de redes sociales estables (Gill, 2000; Olmos,

Fecha recepción: 23-01-19. Fecha de aceptación: 18-05-19

Cristian Abelairas-Gómez

cristianabelairasgomez@gmail.com
2004). Sin embargo, durante la etapa universitaria, se produce un aumento del abandono de un estilo de vida activo (Gómez, Ruiz, García, Granero \& Piéron, 2009) y un descenso de la practica físico-deportiva moderada y vigorosa (Han et al., 2008; Sinclair, Hamlin \& Steel, 2005), convirtiéndose en un momento crítico para la promoción de la actividad física. La reducción de los índices de actividad física parece modificarse de forma dispar en función del sexo, reflejándose este declive en mayor magnitud en las mujeres (Han et al., 2008). Asimismo, la literatura científica ha demostrado que las razones que provocan la práctica en hombres y mujeres son diferentes, atribuyéndose a los hombres motivaciones de carácter más intrínseco y a las mujeres de carácter más extrínsecas durante la etapa universitaria (Egli, Bland, Melton \& Czech, 2011).

El cambio cualitativo y cuantitativo que supone, en cuanto al grado de exigencia y dedicación, el acceso a la universidad produce un punto de inflexión en el estilo de vida de los universitarios, siendo la falta de tiempo para la realización de actividades físico-deportivas la principal causa de abandono durante el periodo universitario (Arce \& De Francisco, 2008; Consejo Superior de Deportes, 2006). Diversos estudios, han analizado el impacto de proyectos experimentales dentro de la comunidad universitaria con el objetivo de incrementar los índices de actividad física (Kim, Lumpkin, Lochbaum, Stegemeier \& Kitten, 2018; Lytle et al., 2017). Sin embargo, la evidencia científica parece demostrar que la implementación de estos programas de actividad física no siempre muestra los niveles de eficacia deseados por la administración (Plotnikoff et al., 2015). De este modo, conocer los motivos intrínsecos y extrínsecos junto con los hábitos de actividad física parece un factor estratégico necesario para tomar decisiones en la planificación de las actividades físico-deportivas propuestas, facilitando así la accesibilidad 
a la práctica y permitiendo generar a la administración una oferta adecuada a las motivaciones e intereses de los estudiantes universitarios de ambos sexos. La etapa universitaria se convierte en una de las más críticas para la continuación de hábitos saludables, destacando entre ellos la práctica de actividad física. Por ello, el conocimiento de los hábitos y motivaciones de los estudiantes universitarios, incluso ya desde la adolescencia (Gálvez, Rodríguez \& Velandrino, 2007) y especialmente en niveles preuniversitarios (HerreraGutiérrez, Brocal-Pérez, Sánchez \& Rodríguez, 2012), se vuelve vital para el futuro desarrollo de programas que permitan fomentar un estilo de vida activo. De manera que, el objetivo de este estudio fue conocer las motivaciones y hábitos de práctica de actividad física en la población universitaria, así como las diferencias existentes entre ambos sexos.

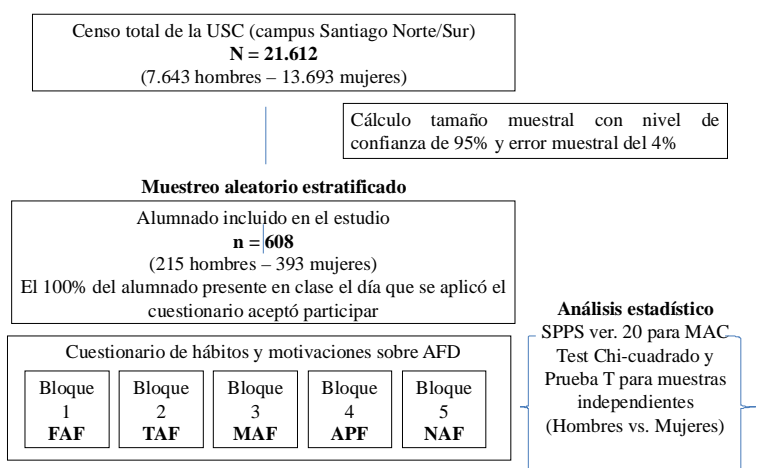

Figura 1. Diagrama de flujo del diseño de la investigación. USC: Universidad de Santiago de Compostela; AFD: Actividad Físico-Deportiva; FAF: Frecuencia y duración de la práctica de Actividad Físico-deportiva; TAF: Tipo de práctica de Actividad Físico-deportiva; MAF: Motivos por los que practica Actividad Físico-deportiva; APF: Abandono de

\section{Material y métodos}

En la Figura 1 se ofrece una representación gráfica del diseño de la investigación con especificación de la población objeto de estudio, la muestra, los grupos y tipo de muestreo, el instrumento de recogida de datos y los análisis estadísticos realizados. Así mismo, se recogen en esta figura los bloques de variables analizados en esta investigación a través de un cuestionario de hábitos y motivaciones sobre actividad físico-deportiva, estos son: (FAF: Frecuencia y duración de la práctica de Actividad Físico-deportiva; TAF: Tipo de práctica de Actividad Físico-deportiva; MAF: Motivos por los que practica Actividad Físico-deportiva; APF:
Abandono de la Práctica Físico-deportiva; NAF: motivos por los que No practica Actividad Físico-deportiva).

\section{Participantes}

La selección de la muestra se realizó utilizando un procedimiento de muestreo aleatorio estratificado por conglomerados (titulación y curso), necesario para garantizar la representatividad de todos los segmentos poblacionales entre los que pudiesen existir diferencias en las variables objeto de estudio. La población universitaria matriculada en la Universidad de Santiago de Compostela en el momento del estudio era de 21.612 alumnos (7.643 hombres y 13.693 mujeres) en un total de 20 centros. La selección de la muestra se realizó utilizando un muestreo aleatorio estratificado por conglomerados (titulación y curso). Para el cálculo del tamaño muestral se eligió un nivel de confianza del 95.5\% y un error muestral del 4\% y se asumió que p=q. Además, se efectuó una afijación proporcional atendiendo al sexo, resultando una muestra compuesta por 608 alumnos, de los cuales 215 eran hombres (35.4\%) y 393 mujeres (64.6\%), con edades comprendidas entre los 18 años y los 61, con una media de 21.45 años y una desviación típica de 3.33 años. El 100\% del alumnado presente en clase el día que se aplicó el cuestionario aceptó cubrirlo (Figura 1). La muestra pertenecía a un total de 11 centros y 17 titulaciones. La distribución de la muestra según cursos académicos fue así: $1^{\circ}(25.3 \%)$ - $2^{\circ}$ (19.4\%) $-3^{\circ}(23.7 \%)-4^{\circ}(17.6 \%)-5^{\circ}(10.2 \%)-$ Máster(3.8\%).

\section{Diseño del estudio}

La recogida de datos fue realizada por un único encuestador, quien con un guion escrito siempre trasmitió a los alumnos encuestados la misma información, con el fin de controlar los sesgos y mantener la calidad de los datos. Los cuestionarios se aplicaron en las aulas disponiendo de toda la duración de la clase para facilitar que los participantes pudiesen responder con tranquilidad y prestando atención a los ítems.

El cuestionario utilizado fue diseñado con el objetivo de recoger información sobre: características del participante, conocimiento de los servicios deportivos universitarios, hábitos físico-deportivos de los sujetos practicantes, aspectos psicosociales de los sujetos que practican actividad físico-deportiva, influencia de la educación física escolar sobre

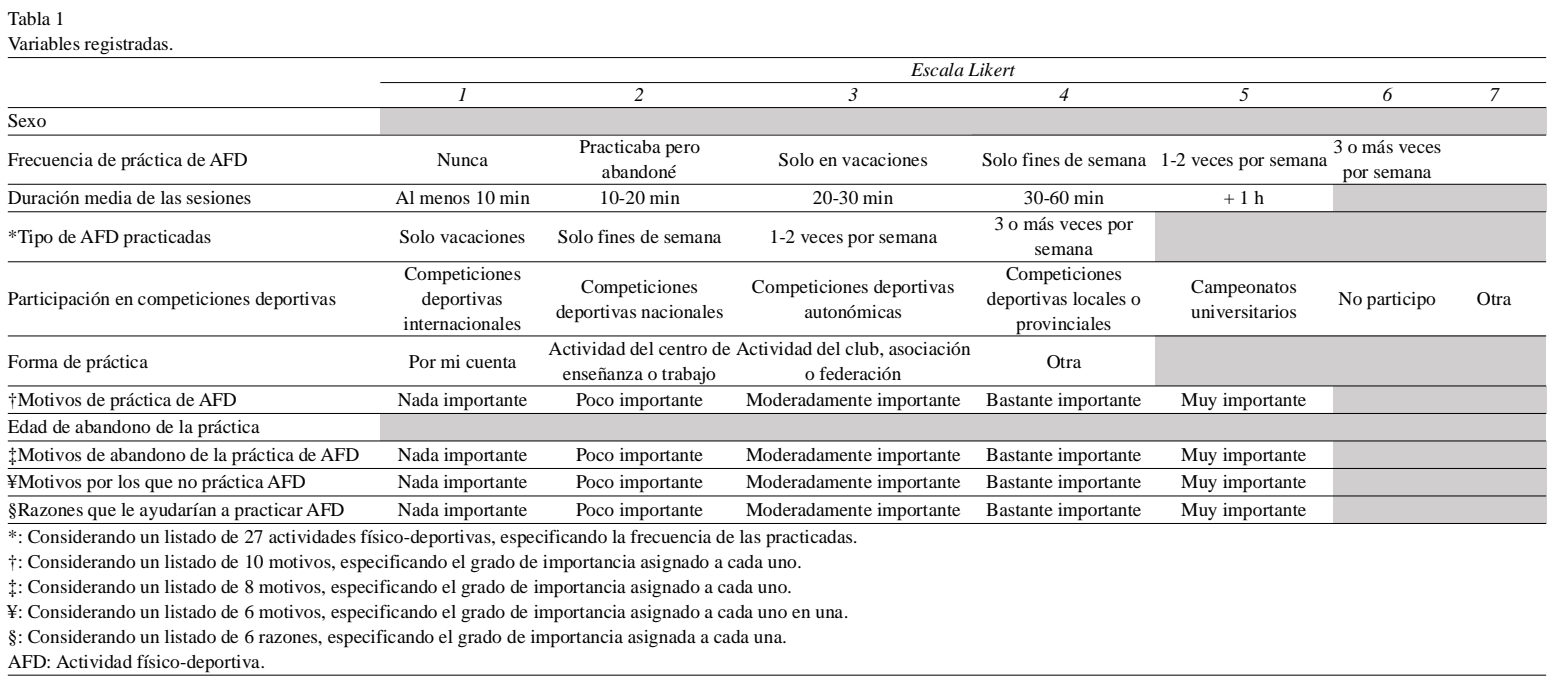


los alumnos con un estilo de vida activo y sedentario, actividades que se desarrollan durante el tiempo libre de la población objetivo y hábitos saludables. No obstante, a efectos de esta investigación solamente se han considerado las variables mostradas en la Tabla 1, muy coincidentes con los ítems del cuestionario original de Castillo, Abad, Giménez y Robles (2012).

\section{Instrumento}

La fuente de información fue de tipo primario, obtenida directamente de los alumnos universitarios a través de un cuestionario. En este caso, se partió de un cuestionario sobre actividad física y estilo de vida de la población universitaria ya validado (Castillo et al. 2012). Mediante consulta a expertos (método Delphi), se adaptó ad hoc para esta investigación. Se utilizó un panel de 10 expertos Licenciados en Ciencias de la Actividad Física y del Deporte y Licenciados en Psicología. Se utilizaron preguntas cerradas y categorizadas, preguntas directas, preguntas de introducción y de filtro, y preguntas de identificación. En esta fase, los expertos revisaron, analizaron y explicaron las posibles mejoras al cuestionario, dando respuesta a un guion prestablecido. Los expertos, desde su visión personal y profesional, valoraron muy positivamente el formato, estructura y los diferentes elementos y aspectos del cuestionario e igualmente aportaron diferentes observaciones, tanto a nivel general como en determinadas preguntas y respuestas del cuestionario, para su mejora y mayor aplicabilidad en el contexto en el que se ha desarrollado esta investigación. Posteriormente, el cuestionario inicial se modificó y completó teniendo en cuenta las sugerencias y mejoras que los expertos propusieron tras su revisión.

Para verificar la comprensión de los ítems que componían el cuestionario definitivo se efectuó una prueba piloto. Se desarrolló en el primer curso del grado en Maestro en Educación Primaria de la Facultad de Ciencias de la Educación y del Deporte de Pontevedra. El objetivo de esta prueba era contrastar si el cuestionario funcionaba como se pretendía, confirmar si se ajustaba a los objetivos de la investigación, detectar posibles errores y realizar las consiguientes mejoras en el procedimiento de toma de datos.

\section{Análisis de los datos}

Para el análisis estadístico se ha utilizado el programa informático SPSS para Macintosh (versión 20.0, Chicago, IL, USA). Primero se realizó un análisis descriptivo de cada variable del cuestionario de forma individualizada (medias, desviaciones típicas, distribuciones de frecuencias), para posteriormente realizar un análisis de relaciones entre variables (test $\dot{\circ}^{2}$ ) y comparaciones entre medias (prueba $t$ para muestras independientes). Se utilizó un nivel de significación de $p$ $<.05$ para todos los análisis estadísticos (Figura 1).

\section{Resultados}

Frecuencia y duración de la práctica de actividad físico-deportiva del alumnado universitario

El 74.35\% de los universitarios practicaban algún tipo de actividad físico-deportiva. Aunque fue mayor en los hombres que en las mujeres, la frecuencia de práctica de activi- dad físico-deportiva entre los alumnos de la universidad de Santiago de Compostela no difiere significativamente entre ambos sexos, $\dot{\div}^{2}(5)=10.56, p=.061$. En la Figura 2 se presenta la frecuencia de práctica físico-deportiva de los alumnos universitarios.

En relación con la duración media de práctica, se ha observado que las mujeres practicaban en su mayoría sesiones de entre 10 minutos y 1 hora, mientras que los hombres se encuentran sobrerrepresentados a partir de 1 hora de práctica. En este caso, la diferencia entre sexos ha alcanzado significatividad estadística, $\stackrel{-2}{(4)}_{(4)}=61.87, p<.001$. No existen asociaciones estadísticamente significativas entre la duración y la frecuencia de la práctica de actividad físico-deportiva, $\div_{(12)}^{2}=18.673, \mathrm{p}=.097$.

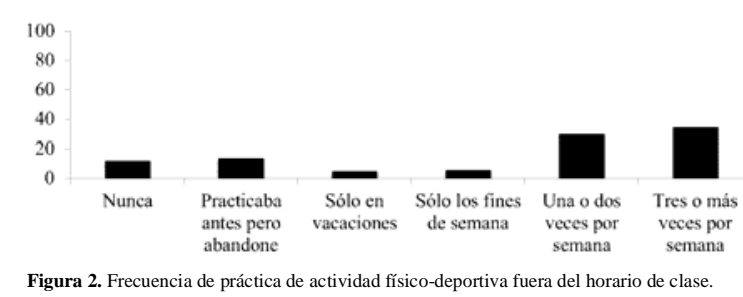

\section{Tipo de práctica de actividad físico-deportiva del alumnado}

Respecto al tipo de actividades físico-deportivas practicadas, tanto los hombres como las mujeres señalaron que las actividades preferidas eran: caminar de forma vigorosa y continuada al menos 10 minutos, jogging, natación, ciclismo y fútbol. Sólo el 22.12\% de los que practicaban algún tipo de actividad físico-deportiva participaban en competiciones deportivas. Se entendía como intensidad vigorosa aquella que provocase una respiración rápida e impidiera mantener una conversación. Del alumnado que competía, el 84\% eran hombres, existiendo diferencias estadísticamente significativas entre sexos en relación con la participación en competiciones deportivas en favor de los hombres, $\stackrel{\circ}{*}_{(7)}=115.406, p$ $<$.001. Los alumnos universitarios compostelanos que practicaban actividad físico-deportiva preferían hacerlo por su cuenta (78.74\%) y en segundo lugar como actividad del club, asociación, organización o federación (16.55\%). La práctica como actividad del centro de enseñanza o de trabajo y otras opciones no presentaron valores relevantes (4.71\% de los practicantes).

\section{Motivos de práctica de actividad físico-deportiva}

En la Tabla 2 se ofrece una relación completa de 10 motivos de práctica de actividad físico-deportiva. Se observaron diferencias estadísticamente significativas entre las medias de ambos sexos en la mayoría de los motivos de práctica. Solamente en los motivos por hacer ejercicio y por evasión no se encontraron diferencias estadísticamente significativas entre hombres y mujeres ( $\mathrm{p}>.05$ en ambos casos). En los motivos por diversión y pasar el tiempo, por encontrarme con amigos/as, porque me gusta la actividad física, porque me gusta competir, por satisfacción personal o porque se me da bien, puntuaron más los hombres que las mujeres. Por su parte, en los motivos por mantener la línea, por mantener y/o mejorar la salud, puntuaron más las mujeres que los hombres. 


\begin{tabular}{|c|c|c|c|c|c|}
\hline Motivo & Sexo & $n$ & Media & $D T$ & $E E$ \\
\hline \multirow{2}{*}{ Por diversión y pasar el tiempo** } & Hombre & 170 & 4.117 & 1.019 & .078 \\
\hline & Mujer & 262 & 3.576 & 1.114 & .069 \\
\hline \multirow{2}{*}{ Por encontrarme con amigos/as** } & Hombre & 168 & 3.381 & 1.222 & .094 \\
\hline & Mujer & 258 & 2.849 & 1.256 & .078 \\
\hline \multirow{2}{*}{ Por hacer ejercicio } & Hombre & 172 & 4.284 & .855 & .065 \\
\hline & Mujer & 268 & 4.332 & .847 & 052 \\
\hline \multirow{2}{*}{ Porque me gusta la actividad física** } & Hombre & 171 & 3.994 & 1.020 & .078 \\
\hline & Mujer & 263 & 3.403 & 1.178 & .073 \\
\hline \multirow{2}{*}{ Por mantener la línea** } & Hombre & 170 & 3.488 & 1.163 & .089 \\
\hline & Mujer & 264 & 3.890 & 1.103 & .068 \\
\hline \multirow{2}{*}{ Por evasión } & Hombre & 167 & 3.335 & 1.301 & .101 \\
\hline & Mujer & 261 & 3.513 & 1.135 & 070 \\
\hline \multirow{2}{*}{ Por mantener $\mathrm{y} / \mathrm{o}$ mejorar la salud** } & Hombre & 170 & 3.917 & 1.074 & .082 \\
\hline & Mujer & 266 & 4.301 & .796 & .049 \\
\hline \multirow{2}{*}{ Porque me gusta competir** } & Hombre & 169 & 3.027 & 1.414 & .109 \\
\hline & Mujer & 257 & 1.665 & .951 & .059 \\
\hline \multirow{2}{*}{ Por satisfacción personal* } & Hombre & 171 & 4.005 & .960 & .073 \\
\hline & Mujer & 265 & 3.766 & 1.062 & 0.065 \\
\hline \multirow{2}{*}{ Porque se me da bien** } & Hombre & 167 & 2.952 & 1.268 & .098 \\
\hline & Mujer & 258 & 2.364 & 1.150 & .072 \\
\hline
\end{tabular}

\section{Abandono de la práctica físico-deportiva}

En la Tabla 3 se ofrece una relación completa de todos los motivos de abandono que figuraban en el cuestionario, siendo a los 18 años (22.7\% de los abandonos), 17 (18.6\% de los abandonos) y 16 (16.5\% de los abandonos) las edades en las que más abandonos se produjeron. En relación con las edades de abandono, no existen diferencias estadísticamente significativas entre sexos, $\stackrel{\circ}{*}_{(14)}=16.352$, p $=$ 292.

En cuanto a los motivos de abandono, no se han encontrado diferencias estadísticamente significativas entre sexos, considerando claramente la mayoría de los alumnos universitarios que la falta de tiempo para estudiar fue el factor más determinante para su abandono (mujeres: $3.765 \pm 1.235$; hombres: $3.435 \pm 1.532$ ). Ninguno de los demás motivos evaluados en esta investigación han llegado a una puntuación de 2 sobre 5, tanto en hombres como en mujeres.

\begin{tabular}{|c|c|c|c|c|c|}
\hline Motivo & Sexo & $n$ & Media & $D T$ & $E E$ \\
\hline \multirow{2}{*}{$\begin{array}{l}\text { Porque mis padres no me dejaron } \\
\text { continuar }\end{array}$} & Hombre & 21 & 1.095 & .436 & .095 \\
\hline & Mujer & 66 & 1.167 & .543 & .067 \\
\hline \multirow{2}{*}{ Porque no tengo tiempo para estudiar } & Hombre & 23 & 3.435 & 1.532 & .320 \\
\hline & Mujer & 68 & 3.765 & 1.235 & 150 \\
\hline \multirow{2}{*}{ Porque mis amigos/as abandonaron } & Hombre & 21 & 1.810 & 1.167 & .255 \\
\hline & Mujer & 66 & 1.970 & 1.403 & 173 \\
\hline \multirow{2}{*}{ Porque cambié de centro de estudios } & Hombre & 21 & 1.810 & 1.537 & .335 \\
\hline & Mujer & 66 & 1.910 & 1.486 & 183 \\
\hline \multirow{2}{*}{ Porque tuve una lesión } & Hombre & 21 & 1.480 & .873 & .190 \\
\hline & Mujer & 67 & 1.432 & 1.033 & .126 \\
\hline \multirow{2}{*}{ Por las exigencias del entrenador/a } & Hombre & 21 & 1.190 & .512 & .112 \\
\hline & Mujer & 66 & 1.230 & .697 & .085 \\
\hline \multirow{2}{*}{ Porque empecé a salir con un chico/a } & Hombre & 21 & 1.381 & 1.071 & .234 \\
\hline & Mujer & 66 & 1.151 & .438 & 054 \\
\hline \multirow{2}{*}{ Porque tuve un hijo/a } & Hombre & 21 & 1.000 & .000 & .000 \\
\hline & Mujer & 63 & 1.079 & .414 & .052 \\
\hline
\end{tabular}

DT: Desviación Típica; EE: Error estándar.

Motivos por los que no practica actividad físico-deportiva el alumnado universitario y factores que le ayudarían a practicar

En la Tabla 4 se ofrece una relación completa de todos los motivos por los que el alumnado no practica y los motivos inhibidores de la no práctica.

Tanto hombres como mujeres fueron coincidentes en la determinación de los principales motivos por los que no practicaban actividad físico-deportiva, esto fue: por no tener tiempo y por pereza o desgana, superando en ambos sexos el 3 de puntuación media. No se han observado diferencias estadísticamente significativas entre las medias de ambos sexos en ninguno de los motivos de influencia.

Respecto a los motivos alegados por el alumnado para iniciar la práctica, hombres y mujeres conincidieron en la valoración de los dos principales factores que les ayudarían a practicar actividad físico-deportiva: tener un horario de actividades compatible con el de clases (4.023 sobre 5 los hombres y 4.418 las mujeres) y tener organizado mi tiempo para estudiar (3.951 los hombres y 4.254 las mujeres). Se observó, no obstante, que todos los motivos señalados en el cuestionario presentaron valoraciones altas -ninguno por debajo de 3-, tanto en el caso de las mujeres como de los hombres. Las mujeres encontraban más importante que los hombres el tener un horario de actividades compatible con el de las clases y tener un monitor/a entrenador/a bien preparado/a $(p<.05$ en ambos casos).

\begin{tabular}{|c|c|c|c|c|c|}
\hline Motivo & Sexo & $n$ & Media & $D T$ & $E E$ \\
\hline \multicolumn{6}{|c|}{ Importancia otorgada a cada motivo por el que no practica } \\
\hline \multirow{2}{*}{ Porque no me gusta } & Hombre & 36 & 2.167 & 1.559 & .260 \\
\hline & Mujer & 111 & 2.045 & 1.246 & .118 \\
\hline \multirow{2}{*}{ Porque no se me da bien } & Hombre & 36 & 1.861 & 1.125 & .188 \\
\hline & Mujer & 109 & 2.046 & 1.235 & .118 \\
\hline \multirow{2}{*}{ Porque no tengo tiempo } & Hombre & 39 & 3.667 & 1.284 & .206 \\
\hline & Mujer & 114 & 3.930 & 1.134 & .106 \\
\hline \multirow{2}{*}{ Porque no hay instalaciones adecuadas } & Hombre & 35 & 2.086 & 1.011 & .171 \\
\hline & Mujer & 107 & 2.224 & 1.049 & .101 \\
\hline \multirow{2}{*}{ Por pereza o desgana } & Hombre & 37 & 3.676 & 1.375 & .226 \\
\hline & Mujer & 113 & 3.725 & 1.197 & .112 \\
\hline \multirow{2}{*}{ Porque no tengo amigos/as con quien hacerlo } & Hombre & 36 & 2.306 & 1.238 & .206 \\
\hline & ${ }^{\circ}$ Mujer & 108 & 2.352 & 1.263 & .121 \\
\hline \multirow{2}{*}{ Por tener una lesión o enfermedad } & Hombre & 2 & 1.000 & .000 & .000 \\
\hline & Mujer & 3 & 3.667 & 2.309 & 1.333 \\
\hline \multicolumn{6}{|c|}{ Importancia de motivos inhibitorios de la no práctica } \\
\hline \multirow{2}{*}{ Tener buenas instalaciones } & Hombre & 41 & 3.658 & 1.132 & .177 \\
\hline & Mujer & 121 & 3.504 & 1.170 & 106 \\
\hline \multirow{2}{*}{ Tener organizado mi tiempo para estudiar } & Hombre & 41 & 3.951 & 1.139 & .178 \\
\hline & Mujer & 122 & 4.254 & .967 & .088 \\
\hline \multirow{2}{*}{ Que los/as amigos/as practiquen también } & Hombre & 41 & 3.195 & 1.100 & .172 \\
\hline & Mujer & 121 & 3.107 & 1.196 & .109 \\
\hline \multirow{2}{*}{$\begin{array}{l}\text { Tener un horario de actividades compatible } \\
\text { con el de clases* }\end{array}$} & Hombre & 43 & 4.023 & 1.123 & .171 \\
\hline & Mujer & 122 & 4.418 & .842 & .076 \\
\hline \multirow{2}{*}{ Que hubiera actividades atractivas } & Hombre & 41 & 3.756 & 9969 & .151 \\
\hline & Mujer & 121 & 4.041 & 1.028 & .093 \\
\hline \multirow{2}{*}{$\begin{array}{l}\text { Tener un monitor/a entrenador/a bien } \\
\text { preparado/a* }\end{array}$} & Hombre & 41 & 3.024 & 1.151 & .180 \\
\hline & Mujer & 122 & 3.566 & 1.239 & .112 \\
\hline
\end{tabular}

\section{Discusión}

Los principales hallazgos de este estudio son: (a) a pesar de que prácticamente tres cuartas partes del alumnado admitió practicar actividad físico-deportiva, apenas una séptima parte de la muestra cumplía con las recomendaciones de la OMS (2010), esto es 150 minutos semanales; (b) se encontraron diferencias entre el porcentaje de práctica de hombres y mujeres, siendo más activos los primeros; (c) caminar de forma vigorosa y continuada al menos 10 minutos se realizaba por más de la mitad del alumnado practicante, casi duplicando a la segunda en número de adeptos; (d) las motivaciones entre hombres y mujeres a la hora de practicar actividad físico-deportiva fueron diferentes.

La transición de la adolescencia a la edad adulta se presenta como un momento angular para adquisición de patrones de comportamiento de un estilo de vida saludable. Según los resultados de esta investigación, el $74.35 \%$ del alumnado universitario practicaba algún tipo de actividad físico-deportiva. Estos resultados son similares a los obtenidos en investigaciones previas de ámbito nacional (Blasco, Capdevila, Pintanel, Valiente \& Cruz, 1996; García-Ferrando y Llopis, 2011; Sánchez Pérez, García Domínguez, Landabaso 
\& De Nicolás y Martínez, 1998). Igualmente, la magnitud de la tasa de actividad física entre el alumnado universitario refleja una tendencia similar a la observada a nivel internacional (Irwin, 2004).

La adquisición de patrones de comportamiento saludable durante la etapa universitaria constituye una estrategia de prevención primaria para la disminución del riesgo de padecer enfermedades no transmisibles (Garett, Brasure, Schmitz, Shultz \& Huber, 2004). Además, la etapa universitaria es comúnmente reconocida como un período crítico para la aparición de desórdenes mentales producidos por los múltiples cambios producidos en el estilo de vida de vida del alumnado universitario (Castillo \& Schwartz, 2013), en ocasiones siendo un factor esencial en el abandono universitario y en el deterioro académico (Keyes, Eisenberg, Perry, Dube, Kroenke \& Dhingra, 2012). Diferentes estudios sugieren que la práctica de actividad física podría tener efectos beneficiosos sobre la salud mental (Janssen \& LeBlanc, 2010; Rosenbaum, Tiedemann \& Ward 2014). Asimismo, un mayor rendimiento académico también se ha relacionado con los niveles de salud, bienestar psicológico y con un estilo de vida activo (Capdevila Seder, Bellmunt Villalonga \& Hernando Domingo, 2015; El Ansari \& Stock, 2010; Santana, Azevedo, Cattuzzo, Hill, Andrade \& Prado, 2017). Además, Pedišiæ, Greblo, Phongsavan, Milton y Bauman, (2015) demostraron que valores elevados de bienestar se presentan en el alumnado universitario físicamente activo. Consecuentemente, las políticas de promoción de hábitos saludables y de bienestar por parte de las entidades universitarias son fundamentales para mejorar la salud física, mental y las tasas de rendimiento académico de la población universitaria. Debe abandonarse la percepción de que la universidad es un estamento puramente formativo, reconociendo que es un entorno educativo privilegiado para la promoción de la salud con una gran relevancia sociosanitaria (Lozano Polo et al. 2013).

Si diferenciamos por sexos, en esta investigación el 80\% de los hombres realizaban actividades físico-deportivas, frente a un $71.25 \%$ del alumnado universitario femenino. En otra investigación sobre la actitud de los universitarios murcianos ante la práctica físico-deportiva han detectado que el 75.2\% de los hombres practicaba algún tipo de actividad físicodeportiva, mientras que entre las mujeres esta tasa descendía hasta el 61.3\% (Lores \& Murcia, 2008). Una mayor discrepancia se ha encontrado entre los resultados obtenidos en este estudio y una investigación con universitarios de Valencia, la cual ha concluido que sólo el 5.6\% del alumnado masculino no practicaba deporte, mientras que en el caso de las mujeres este porcentaje subía hasta el 49\% (MolinaGarcía, Castillo \& Pablos, 2007). Por su parte, Allende, Juan y Montes (2009) concluyeron que, entre el alumnado universitario, eran los hombres (65.3\%) quienes eran más participativos. Además, respecto a los que afirmaron no haber realizado nunca y abandonado la práctica físico-deportiva, fueron las alumnas quienes presentaron los índices más elevados de inactividad (7.1\% frente al 2.8\% que nunca ha realizado y $44.9 \%$ frente al $31.9 \%$ en abandono).

Según la OMS (2010), los adultos de 18 a 64 años de edad deberían realizar un mínimo de 150 minutos semanales de práctica de actividad física aeróbica de intensidad moderada, 75 minutos de actividad física aeróbica vigorosa, o una combinación equivalente de actividades moderadas y vigorosas. La actividad física realizada por los individuos se deberá extender durante un período de tiempo de 10 minutos como mínimo. A pesar, de que un 74.35\% de los estudiantes universitarios afirmaron practicar actividad física, los resultados muestran que tan sólo un 15\% cumplía con la líneaguía sobre actividad física propuesta por la OMS (2010). Por su parte, Práxedes, Sevil, Moreno, del Villar y García-González (2016) encontraron que más de la mitad de los estudiantes universitarios que participaron en su estudio se situaban por debajo de los índices de actividad física recomendados. Roux et al. (2008) advirtieron que la mayor parte de la población no conoce las líneas-guía sobre actividad física, reduciendo de este modo la efectividad de los períodos dedicados a su práctica. Según Kwan et al. (2002) la implementación de campañas comunitarias (i.e. notas de prensa, radio, correos directos, vallas publicitarias, cartelería) por parte de los poderes públicos y otros agentes, podría contribuir a la difusión de las recomendaciones de la OMS (2010) sobre actividad física. Asimismo, esta estrategia informativa debería encontrarse secundada por grupos de autoayuda, donde se asesore a los estudiantes sobre factores de riesgo y efectos positivos de la actividad física sobre la salud.

En nuestro estudio, el tipo de actividad física más frecuentemente desarrollada por la población universitaria fue caminar de forma vigorosa y continuada durante al menos 10 minutos. Los resultados obtenidos en cuanto al tipo de actividad realizada contrastan con estudios previos en el ámbito universitario (Castillo, 2006; Lores \& Murcia, 2006; Pliego, Díaz de León, Robles \& Celis, 2007; Sánchez Pérez et al., 1998). Las diferencias encontradas con estudios previos podrían encontrar su motivo causal en los criterios utilizados durante la elaboración de los cuestionarios, no apareciendo entre las opciones de respuesta el caminar de forma vigorosa durante al menos 10 minutos en otros estudios. Por su parte, la OMS (2010) señala que toda actividad aeróbica mantenida durante un intervalo de tiempo de al menos 10 minutos es considerada como actividad física, lo que muestra que puede ser interesante su incorporación como posible tipo de actividad físico-deportiva en estos estudios. A este respecto, Horacek et al. (2018) analizaron el efecto de la accesibilidad peatonal y en bicicleta de los estudiantes a las instalaciones universitarias, observando una asociación entre la facilidad de acceso y la reducción del índice de masa corporal. Por tanto, facilitar la accesibilidad peatonal y en bicicleta a instalaciones y centros universitarios podría ser una importante medida a tener en cuenta para la promoción de la práctica de actividad física. Además, los centros podrían modificar las actitudes desde un enfoque informativo mediante carteles que traten de alentar al individuo a la realización de actividad física dentro de las instalaciones universitarias, como por ejemplo la elección del uso de una escalera en detrimento del ascensor (Kwan et al. 2002). En nuestra investigación, el alumnado universitario consideró que los motivos que más inciden en la práctica de actividad físicodeportiva son por este orden: por hacer ejercicio físico, por mantener y/o mejorar la salud, por satisfacción personal, por diversión y pasar el tiempo, porque les gusta la actividad físico-deportiva y por evasión/escapar de la rutina. Por su parte, los motivos menos relevantes fueron porque 
les gustaba competir y por encontrarse con amigos. Estos resultados son similares a los obtenidos por una encuesta de hábitos deportivos desarrollada en la población española durante el año 2010, la cual reveló que los principales motivos por los que la población española practica actividad físico-deportiva son por hacer ejercicio físico, por diversión y pasar el tiempo, por mantener y/o mejorar la salud, y porque le gusta el deporte (García-Ferrando \& Llopis, 2011). En este caso la competición y encontrarse con amigos tampoco fueron factores determinantes para la práctica (GarcíaFerrando \& Llopis, 2011).

En relación con el sexo, al igual que en esta investigación, estos autores también han encontrado diferencias estadísticamente significativas entre hombres y mujeres en cuanto a los motivos de práctica. En un estudio realizado con el alumnado de las tres universidades gallegas se obtuvieron resultados similares señalando que las tres principales motivaciones para la práctica de los universitarios gallegos son las vertientes: «lúdica» (44\%), «salud» (35\%) y «competir» (8\%) (Fernández \& Soidán, 2010). En consecuencia, el conocimiento de los motivos de práctica y de las diferencias existentes entre ambos sexos, preferentemente ya desde la adolescencia como sugieren Gálvez, Rodríguez y Velandrino (2007) y especialmente en niveles preuniversitarios (Herrera-Gutiérrez et al. 2012), supone un factor esencial para crear estrategias motivacionales para adherir a los integrantes de la comunidad universitaria que afirman no llevar un estilo de vida activo. De esta forma, conocer las motivaciones individuales que llevan a los estudiantes universitarios a la realización de práctica físico-deportiva constituye la base para la implementación de políticas eficaces en la promoción de la actividad física (Deliens, Deforche, De Bourdeaudhuij \& Clarys, 2015; Herazo, Hernández \& Domínguez, 2012; Leyton Román, García Matador, Fuentes García \& Jiménez Castuera, 2018). El motivo más alegado por la población universitaria es practicar para mejorar la salud, por lo que en futuras investigaciones podría ser interesante analizar si los estudiantes que aseguran no practicar es por desconocimiento o falta de información de los beneficios que supone un estilo de vida activo.

La etapa universitaria se postula como un período transcendental para la adherencia a la práctica de actividad física. En esta línea, los resultados obtenidos muestran cómo la edad en la que se produce una mayor tasa de abandono es a los 18 (22.7\% de los abandonos), seguido de los 17 (18.6\% de los abandonos) y los 16 años (16.5\% de los abandonos), situándose la media de edad en 18.42 años de edad. Así mismo, el presente estudio muestra que el motivo determinante para el abandono de la práctica físico-deportiva es la falta de tiempo para estudiar, coincidiendo este hallazgo con los encontrados por otros autores (Castillo, 2006; GarcíaFerrando \& Llopis, 2011; Fernández \& Soidán, 2010; Lores \& Murcia, 2008; Pliego et al., 2007). A este respecto, los estudiantes universitarios señalaron que tener un horario compatible con el de clases podría favorecer el iniciarse o retomar de nuevo la práctica deportiva. Por ello, el crear una oferta adaptada a los horarios de la población universitaria parece determinante para incrementar los niveles de práctica y sus beneficios dentro de los integrantes de la comunidad universitaria. Con el fin de resolver los motivos para no prac- ticar, las universidades podrían implementar políticas basadas en la modificación de la estructura de los entornos físicos del campus con el fin de generar una mayor accesibilidad caminando y en bicicleta (Horacek et al., 2018), creación de rutas a pie en el interior de los centros universitarios (AbilleiraGonzález, Fernández-Villarino, Varela-Casal, Arufe-Giráldez, Silva-Piñeiro \& González-González, 2018; Gilson, McKenna \& Cooke, 2008) y apoyo telemático de personal deportivocualificado (Opdenacker \& Boen, 2008). Además, todas las iniciativas llevadas a cabo deben prestar especial atención a las mujeres debido a los bajos niveles de actividad física mostrados en diferentes estudios (Práxedes et al. 2016). Futuras investigaciones podrían analizar el efecto de este tipo de políticas dentro de la comunidad universitaria.

\section{Aplicaciones Prácticas}

Los hallazgos encontrados en esta investigación pueden ser de utilidad para los Servicios Deportivos Universitarios de cara a posibles acciones de fomento de la actividad físico-deportiva entre el alumnado. A la vista de los resultados obtenidos y teniendo en cuenta la evidencia científica, un análisis de los intereses y motivaciones de los estudiantes es un buen punto de partida para el diseño de intervenciones que tengan como objetivo desarrollar acciones que favorezcan la adquisición del hábito de práctica físico-deportiva y su adherencia a largo plazo.

Políticas que pongan el énfasis en una mejor estructuración del tiempo de estudio por parte del alumnado y una conciliación de la práctica físico-deportiva con los horarios de clases, haciendo especial hincapié en los cursos iniciales de los estudios universitarios, podrían ser factores relevantes a tener en cuenta en la planificación de la oferta de los servicios deportivos universitarios.

Como perspectiva futura, sería interesante ampliar el objeto de estudio sobre una muestra mayor y de diferentes zonas geográficas para analizar la posible influencia del entorno próximo en los hábitos de actividad físico-deportiva. Además, sería conveniente analizar la oferta físico-deportiva universitaria para evaluar si ésta responde a las necesidades y demandas del alumnado universitario.

\section{Conclusiones}

El objetivo principal del presente estudio fue conocer las motivaciones y hábitos de práctica de actividad física en la población universitaria, así como conocer las diferencias existentes entre ambos sexos. Las conclusiones de este estudio son: (a) alrededor del 75\% del alumnado universitario practicaba algún tipo de actividad físico-deportiva, (b) la actividad «caminar de forma vigorosa y continuada al menos 10 minutos» fue la que más adeptos aglutinó, no existiendo diferencias estadísticamente significativas entre sexos en la frecuencia de práctica, (c) aproximadamente el 85\% del alumnado que practicaba actividad físico-deportiva le dedicaba más de 20 minutos a cada sesión, y concretamente casi el 37\% destinaba 1 hora o más, (d) hacer ejercicio físico fue el principal motivo de práctica esgrimido por la mayoría de sujetos de ambos sexos, (e) la edad media de abandono de la práctica físico-deportiva fue a los 18.42 años y los motivos más seña- 
lados por el alumnado masculino y femenino es la falta de tiempo para estudiar.

\section{Referencias}

Abilleira-González, M., Fernández-Villarino, M., Varela-Casal, C., Arufe-Giráldez, V., Silva-Piñeiro, R., \& GonzálezGonzález, S. (2019). Physical activity intervention program through walking routes in sedentary university students. Journal of Human Sport and Exercise,14(2), doi: https:/ /doi.org/10.14198/jhse.2019.142.13

Allende, G.F., Juan, F. R., \& Montes, M. E. G. (2009). Relación de algunos correlatos biológicos y demográficos con la práctica físico-deportiva en estudiantes universitarios. El caso de la Universidad de Guadalajara, México. Revista Internacional de Ciencias del Deporte, 5(14), 59-80.

Arce, C. \& De Francisco, C. (2008). A realidade da práctica deportiva en Galicia. Santiago de Compostela: Universidade de Santiago de Compostela.

Ato, M.; López, J.J.; Benavente, A. (2013). Un sistema de clasificación de los diseños de investigación en psicología. Anales de Psicología, 29(3), 1038-1059.

Blasco, T., Capdevila, L.L., Pintanel, M., Valiente, L. \& Cruz, J. (1996). Evolución de los patrones de actividad física en estudiantes universitarios. Revista de Psicología del Deporte, 5(2), 51-63.

Bull, F.C., Gauvin, L., Bauman, A., Shilton, T., Kohl, H.W. \& Salmon, A. (2010). The Toronto Charter for Physical Activity: A Global Call for Action. Journal of Physical Activity \& Health, 7(4), 421-422.

Capdevila Seder, A., Bellmunt Villalonga, H., \& Hernando Domíngo, C. (2015). Estilo de vida y rendimiento académico en adolescentes: comparación entre deportistas y no-deportistas. Retos: Nuevas Tendencias en Educación Física, Deportes y Recreación, 27, 28-33.

Castillo, E. (2006). Hábitos de práctica de actividad física y estilo de vida saludable del alumnado de la Universidad de Huelva (Tesis doctoral). Recuperado de http:// rabida.uhu.es/dspace/handle/10272/2644

Castillo, L.G. \& Schwartz, S.J. (2013). Introduction to the special issue on college student mental health. Journal of Clinical Psychology, 69(4), 291-297.

Castillo, E., Abad, M.T., Giménez, F.J. \& Robles, J. (2012). Diseño de un cuestionario sobre hábitos de actividad física y estilo de vida a partir del método Delphi. EBalomnano.com: Revista de Ciencias del Deporte, 8(1), 51-66.

Consejo Superior de Deportes (2006). El Modelo del Deporte Universitario Español. Córdoba: Universidad de Córdoba.

Deliens, T., Deforche, B., De Bourdeaudhuij, I. \& Clarys, P. (2015). Determinants of physical activity and sedentary behaviour in university students: a qualitative study using focus group discussions. BMC Public Health, 15(1), 201-210.

Egli, T., Bland, H. W., Melton, B. F. \& Czech, D.R. (2011). Influence of age, sex, and race on college students' exercise motivation of physical activity. Journal of American College Health, 59(5), 399-406.

El Ansari, W. \& Stock, C. (2010). Is the health and wellbeing of university students associated with their academic performance? Cross sectional findings from the United Kingdom. International Journal of Environmental Research and Public Health, 7(2), 509-527.

Fernández, D.A. \& Soidán, J.L.G. (2010). Motivación hacia la práctica físico-deportiva de universitarios gallegos. $R e$ vista de Investigación en Educación, 8, 128-138.

Gálvez, A., Rodríguez, P.L. \& Velandrino, A. (2007). Influencia de determinados motivos de práctica físico-deportiva sobre los niveles de actividad física habitual en adolescentes. Cuadernos de Psicología del Deporte, 7(1), 7184.

García-Ferrando, M. \& Llopis, R. (2011). Ideal democrático y bienestar personal. Encuesta sobre los hábitos deportivos en España 2010. Madrid: Centro de Investigaciones Sociológicas-Consejo Superior de Deportes.

Garrett, N.A., Brasure, M., Schmitz, K.H., Schultz, M.M. \& Huber, M.R. (2004). Physical inactivity: direct cost to a health plan. American Journal of Preventive Medicine, 27(4), 304-309.

Gill, D.L. (2000). Psychological dynamics of sport and exercise. Champaign, Il: Human Kinetics.

Gilson, N., McKenna, J. \& Cooke, C. (2008). Experiences of route and task-based walking in a university community: qualitative perspectives in a randomized control trial. Journal of Physical Activity and Health, 5(1), 176-182.

Gómez, M., Ruiz, F., García, M., Granero, A. \& Piéron, M. (2009). Motivaciones aludidas por los universitarios que practican actividades físico-deportivas. Revista Latinoamericana de Psicología, 41(3), 519-532.

Han, J.L., Dinger, M.K., Hull, H.R., Randall, N.B., Heesch, K.C. \& Fields, D.A. (2008). Changes in women's physical activity during the transition to college. American Journal of Health Education, 39(4), 194-199.

Herazo, Y., Hernández, J. \& Domínguez, R. 2012. Etapas de cambio y niveles de actividad física en estudiantes universitarios de Cartagena (Colombia). Salud Uninorte, 28(2), 298-307.

Herrera-Gutiérrez, E., Brocal-Pérez, D., Sánchez, D. J. \& Rodríguez, J. M. (2012). Relación entre actividad física, depresión y ansiedad en adolescentes. Cuadernos de Psicología del Deporte, 12(2), 31-38.

Herrera-Gutiérrez, E., Olmos-Soria, M. \& Brocal-Pérez, D. (2015). Efectos psicológicos de la práctica del Método Pilates en una muestra universitaria. Anales de Psicología, 31(3), 916-920.

Horacek, T.M., Dede Yildirim, E., Kattelmann, K., Brown, O., Byrd-Bredbenner, C., Colby, S. \& Morrell, J. (2018). Path analysis of campus walkability/bikeability and college students' physical activity attitudes, behaviors, and body mass index. American Journal of Health Promotion, 32(3), 578-586.

Irwin, J.D. (2004). Prevalence of university students' sufficient physical activity: a systematic review. Perceptual and Motor Skills, 98(3), 927-943.

Janssen, I. \& LeBlanc, A.G. (2010). Systematic review of the health benefits of physical activity and fitness in schoolaged children and youth. International Journal of Behavioral Nutrition and Physical Activity, 7(1), 40. Joseph, R.P., Royse, K.E., Benitez, T.J. \& Pekmezi, D.W.(2014). 
Physical activity and quality of life among university students: exploring self-efficacy, self-esteem, and affect as potential mediators. Quality of Life Research, 23(2), 659-667.

Keyes, C.L., Eisenberg, D., Perry, G.S., Dube, S.R., Kroenke, K. \& Dhingra, S.S. (2012). The relationship of level of positive mental health with current mental disorders in predicting suicidal behavior and academic impairment in college students. Journal of American College Health, 60(2), 126-133.

Kim, Y., Lumpkin, A., Lochbaum, M., Stegemeier, S. \& Kitten, K. (2018). Promoting physical activity using a wearable activity tracker in college students: A cluster randomized controlled trial. Journal of Sports Sciences, 1-8.

Kwan, M.Y., Cairney, J., Faulkner, G.E. \& Pullenayegum, E.E. (2012). Physical activity and other health-risk behaviors during the transition into early adulthood: a longitudinal cohort study. American Journal of Preventive Medicine, 42, 14-20.

Lees, S.J. \& Booth, F.W. (2004). Sedentary death syndrome. Canadian Journal of Applied Physiology, 29(4), 447460.

Leyton Román, M., García Matador, J., Fuentes García, J. P., \& Jiménez Castuera, R. (2018). Análisis de variables motivacionales y de estilos de vida saludables en practicantes de ejercicio físico en centros deportivos en función del género. Retos: Nuevas Tendencias en Educación Física, Deportes y Recreación, 34, 166-171.

Lores, A. P., \& Murcia, J.A.M. (2008). Actitud de los universitarios ante la práctica físico-deportiva: diferencias por géneros. Revista de Psicología del Deporte, 17(1), 7-23.

Lores, A.P., \& Murcia, J.A.M. (2006). Características de la práctica físico-deportiva en estudiantes universitarios. Conexöes, 4(1), 125-151.

Lozano Polo, A., Herrera-Gutiérrez, E., Martínez Moreno, F., Gutiérrez García, J., Brocal-Pérez, D., Árense Gonzalo, J., ... Torres Chacón, M. D. (2013). Estudio de Factores Relacionados con la Salud en el Alumnado Universitario ( $1^{\text {a }}$ ed). Murcia; Consejería de Sanidad y Política Social, Región de Murcia.

Lytle, L.A., Laska, M.N., Linde, J.A., Moe, S.G., Nanney, M.S., Hannan, P.J., \& Erickson, D. J. (2017). Weight-gain reduction among 2-year college students: the CHOICES RCT. American Journal of Preventive Medicine, 52(2), 183-191.

Molina-García, J., Castillo, I. \& Pablos, C. (2007). Bienestar psicológico y práctica deportiva en universitarios. Motricidad. European Journal of Human Movement, 18, 79-91.

Montero, I. \& León, O.G. (2007). A guide for naming research studies in psychology. International Journal of Clinical and Health Psychology, 7(3), 847-862

Olmos, J.G. (2004). La actividad deportiva como instrumento y agente de formación académica en la educación superior universitaria. Revista de Educación, 335, 95-103.

Opdenacker, J. \& Boen, F. (2008). Effectiveness of face-toface versus telephone support in increasing physical activity and mental health among university employees. Journal of Physical Activity and Health, 5(6), 830-843.

Organización Mundial de la Salud (2010). Recomendaciones
Mundiales de actividad física para la salud. Ginebra: Organización Mundial de la Salud.

Pedišiæ, •., Greblo, Z., Phongsavan, P., Milton, K., \& Bauman, A.E. (2015). Are total, intensity-and domain-specific physical activity levels associated with life satisfaction among university students? Plos One, 10(2), 1-21.

Pliego, A., Díaz de León, M., Robles, M. \& Celis, R. (2007). Hábitos de actividad física en la comunidad universitaria del instituto tecnológico de estudios superiores de occidente (ITESO). Revista Brasileira de Ciencia e Movimento, 15(4), 67-72.

Plotnikoff, R.C., Costigan, S.A., Williams, R.L., Hutchesson, M.J., Kennedy, S.G., Robards, S.L. \& Germov, J. (2015). Effectiveness of interventions targeting physical activity, nutrition and healthy weight for university and college students: a systematic review and meta-analysis. International Journal of Behavioral Nutrition and Physical Activity, 12(1), 45.

Powell, K.E. \& Blair, S.N. (1994). The public-health burdens of sedentary living habits-theoretical but realistic estimates. Medicine \& Science in Sport \& Exercise, 26(7), 851-856.

Práxedes, A., Sevil, J., Moreno, A., del Villar, F. \& GarcíaGonzález, L. (2016). Niveles de actividad física en estudiantes universitarios: diferencias en función del género, la edad y los estados de ánimo. Revista Iberoamericana de Psicología del Ejercicio y el Deporte, 11(1), 123-132.

Rosenbaum, S., Tiedemann, A. \& Ward, P.B. (2014). MetaAnalysis Physical Activity Interventions for People With Mental Illness: A Systematic Review and Meta-Analysis. Journal of Clinical Psychiatry, 75(0), 1-11.

Roux, L., Pratt, M., Tengs, T. O., Yore, M.M., Yanagawa, T.L., Van Den Bos, J. \& Kohl, H.W. (2008). Cost effectiveness of community-based physical activity interventions. American Journal of Preventive Medicine, 35(6), 578588.

Sánchez Pérez, A., García Domínguez, F., Landabaso, V., \& De Nicolás y Martínez, L. (1998). Participación en actividad física de una muestra universitaria a partir del modelo de las etapas de cambio en el ejercicio físico: un estudio piloto. Revista de Psicología del Deporte, 7(2), 233245.

Santana, C.C.A., Azevedo, L.B., Cattuzzo, M.T., Hill, J.O., Andrade, L.P. \& Prado, W.L. (2017). Physical fitness and academic performance in youth: A systematic review. Scandinavian Journal of Medicine \& Science in Sports, 27(6), 579-603.

Sinclair, K.M., Hamlin, M.J. \& Steel, G (2005). Physical activity levels of first-year New Zealand university students: a pilot study. Youth Studies Australia, 24(1), 38-42.

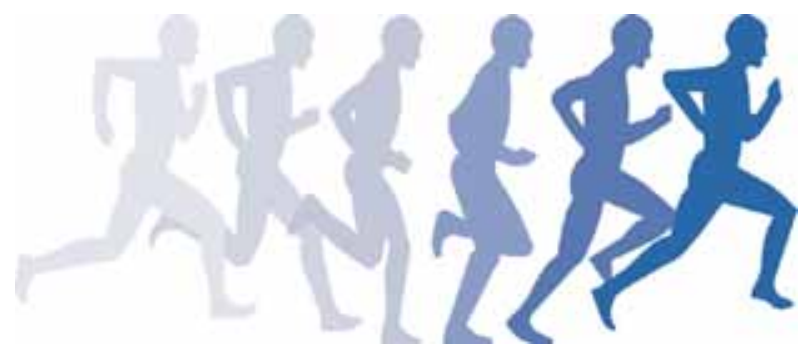

